\title{
A small world full of opportunities
}

\author{
Light-concentration effects in photonic nanostructures, reviewed in this issue, promise new applications \\ ranging from tumour therapy to catalysis and enhanced solar cells.
}

The effects of surface plasmons on optical properties can be astounding. Some of the most beautiful examples of plasmonic effects aren't even to be found in modern research labs. A visit to a museum or medieval cathedrals is all it takes, as medieval glass blowers inadvertently used metallic nanostructures with strong plasmonic effects. Prominent examples of their art are the Lycurgus cup in the British Museum in London and some stained-glass windows in cathedrals such as the rose window in Notre Dame de Paris.

The effects are based on local oscillations of electron plasmas at the surfaces of metals, so-called surface plasmons. What makes plasmonics such an attractive field of research is the ability of surface plasmons to interact with light at wavelengths much longer than the size of those nanostructures and consequently the capability to modify and manipulate the local light fields.

"Plasmonics has given to photonics the ability to go to the nanoscale and properly takes its place among the nanosciences," is how Harry Atwater, a physicist from California Institute of Technology, describes it $^{1}$.

The possibilities in nanophotonic applications are plenty. In this issue of Nature Materials we take a closer look at some of the intriguing functionalities emerging out of light-concentration effects in plasmonic nanostructures. A broad overview on the topic is presented on page 193 by Mark Brongersma and colleagues ${ }^{2}$. For example, these nanostructures could be of interest for enhanced near-field scanning optical microscopes, and for nonlinear optics, where enhanced optical fields could be used to generate extreme-ultraviolet radiation. The concentration of optical fields in small volumes supported by surface plasmons is also studied for spasers, surfaceplasmon-based nanoscale lasers. Spasers may become important as subwavelength sources of coherent light.

Purely optical effects are only one aspect highlighted in the review. Plasmonic devices can also be used to create thermal effects, because under light illumination the nanostructures heat-up locally owing to absorption in the metal structures. Indeed, heating effects are already under investigation as a treatment for cancer, where tumour cells are destroyed by plasmonic nanoparticles excited by an infrared laser beam. Furthermore, photothermal effects could be used to control chemical reactions in the vicinity of plasmonic structures, particularly as many metal surfaces act as catalysts.

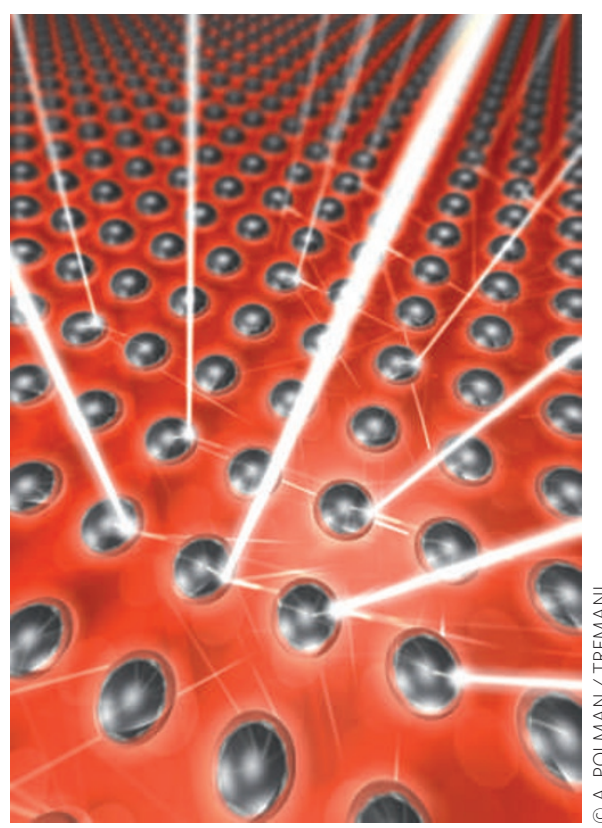

Light absorption in semiconductor solar cells. The silver nanoparticles redirect incoming light and focus it into the semiconductor for higher efficiency. Note that the white light beams are only a schematic depiction; actual optical fields on the nanoscale would appear far more complex.

Last but not least, an application that has gained considerable commercial interest is the use of plasmonic nanostructures to boost the efficiency of solar cells. This is the topic of a separate, in-depth review by Harry Atwater and Albert Polman ${ }^{3}$. There are different ways in which plasmonics can function in solar-cell devices. One obvious method is to concentrate light into the semiconductor layer. The higher intensities of light in the active layer then lead to disproportionate increases in absorption efficiency. In addition, more recent plasmonic structures use a different approach: they take the vertically incoming light and guide it in the horizontal direction along the semiconductor film, thereby maximizing light-matter interaction.

Although such concepts could of course be used to enhance overall efficiency of solar cells, the benefit of such schemes may rather lie in the fact that one could fabricate photovoltaic devices that use much less active material while achieving the same efficiency. This not only leads to a costeffective design but more importantly uses much less material. As Atwater and Polman argue, many essential elements used in a number of photovoltaic device designs, such as tellurium or indium, are not sufficiently abundant to support a significant expansion in solar-cell production.

Obviously, sophisticated light management in solar cells is subject to intensive investigations in itself, and there are a number of design suggestions that do not rely on plasmonic effects. One approach is to use microrod arrays in between which light is scattered. As Michael Kelzenberg and colleagues demonstrate in this issue ${ }^{4}$, solar-cell devices that use only $1 \%$ of the active material of conventional photovoltaic cells are capable of achieving almost similar light-conversion efficiencies.

The plasmonic applications presented in this issue are merely a small aspect of nanophotonics. More complex designs with advanced functionality might emerge in the future. For example, in metamaterials the negative dielectric permittivity near the plasmon resonance is essential for devices at visible frequencies. The design flexibility of metamaterials towards a desired functionality may point towards the possible use of composite structures also for plasmonic applications. Another nascent area that is the focus of a special session at this year's March meeting of the American Physical Society is quantum plasmonics, which emerges in the limit of closely coupled metallic structures.

However the field of plasmonics will evolve, the reviews that form part of this focus issue present a glimpse on a promising area of research where there is still plenty to be discovered. Stay tuned.

\footnotetext{
References

1. Heber, J. Nature 461, 720-722 (2009)

2. Schuller, J. A. et al. Nature Mater. 9, 193-204 (2010)

3. Atwater, H. A. \& Polman, A. Nature Mater. 9, 205-213 (2010).

4. Kelzenberg, M. D. et al. Nature Mater. 9, 239-244 (2010).
} 